\title{
CIENCIA Y TECNOLOGÍA COMO MOTOR DEL DESARROLLO ECONÓMICO Y SOCIAL DEL ECUADOR
}

\author{
AUTORES: Luis Fernando Lucio Villacreses ${ }^{1}$ \\ Miriam Adriana Castillo Merino² \\ Alfredo Lesvel Castro Landin ${ }^{3}$ \\ Robards Javier Lima Pisco ${ }^{4}$
}

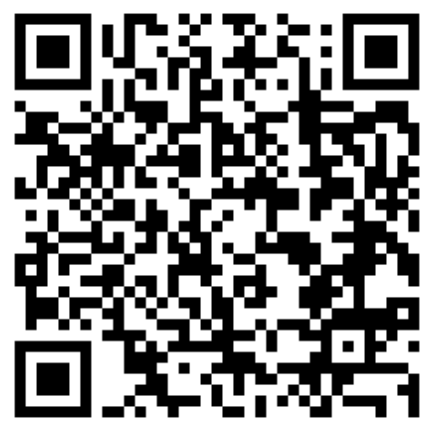

\section{DIRECCIÓN PARA CORRESPONDENCIA:luis.lucio@unesum.edu.ec}

Fecha de recepción: 105/04/2020

Fecha de aceptación: 30/04/2020

RESUMEN

El presente artículo muestra una revisión histórica de la inversión que ha realizado el estado ecuatoriano en Ciencia y Tecnología, con el objeto de mostrar resultados cualitativos de este proceso. La metodología utilizada fue exploratoria y descriptiva. Para este cometido se revisaron fuentes oficiales, se tomaron datos estadísticos, económicos y referencias que describen la inversión realizada en nuestro país. En este contexto, el gobierno del Ec. Rafael Correa, entre los años 2007 - 2017 incrementó progresivamente la inversión. Para el último año en referencia la Secretaría de Educación Superior, Ciencia y Tecnología - SENESCYT presentó una ejecución presupuestaria de las Instituciones de Educación Superior de 1.199 millones de USD equivalente al $0,46 \%$ del Producto Interno Bruto, beneficiándose un total de 59 universidades y escuelas politécnicas y 241 institutos técnicos y tecnológicos. Para el año 2018 el porcentaje de inversión se mantuvo con el gobierno del presidente Lenin Moreno, y con la finalidad de cumplir con los Objetivos de Desarrollo Sostenible, Meta 8.2: Lograr niveles más elevados de productividad mediante

\footnotetext{
${ }^{1}$ Ing. Forestal, Magister en Educación y Desarrollo Social, Docente de la Carrera de Ingeniería Ambiental de la Universidad Estatal del Sur de Manabí. Jipijapa, Manabí, Ecuador. luis.lucio@unesum.edu.ec ORCID ID: https://orcid.org/0000-0002-3757-7183

${ }^{2}$ Ing. en Computación y Redes. Magister en Sistema de Información Gerencial. Docente de la Carrera de Tecnologías de la Información de la Universidad Estatal del Sur de Manabí Jipijapa, Manabí, Ecuador. miriam.castillo@unesum,edu.ec

${ }^{3}$ Ing. Agrónomo. Master en Ciencias Agrícolas, Docente en la carrera de Ing. Ambiental. Jipijapa, Manabí, Ecuador. afredolandin.55@gmail.com

${ }^{4}$ Economista, Magister en Administración Ambiental. Docente de la Carrera de Tecnologías de la Información de la Universidad Estatal del Sur de Manabí. Jipijapa, Manabí, Ecuador. robardslima@ gmail.com
} 
Luis Fernando Lucio Villacreses, Miriam Adriana Castillo Merino, Alfredo Lesvel Castro Landin...

la modernización tecnológica y la innovación, inicia en junio de 2019 la construcción del Plan Nacional de Ciencia, Tecnología, Innovación y Saberes Ancestrales 2030. A la presente fecha se puede indicar que uno de los resultados encontrados indica que nuestro país carece de una guía filosófica y una programación estratégica, integradora, generada con la participación de los sectores vitales del país para iniciar el salto requerido en investigación y desarrollo y promover ciencia, tecnología e innovación, y el crecimiento económico del país.

PALABRAS CLAVE: ciencia; desarrollo; innovación; investigación; tecnología

\section{CIENCE AND TECHNOLOGY AS A DRIVER OF ECUADOR'S ECONOMIC AND SOCIAL DEVELOPMENT}

\section{ABSTRACT}

This article shows a historical review of the investment that the Ecuadorian state has made in Science and Technology, in order to show qualitative results of this process. The methodology used was exploratory and descriptive. For this comet, official sources were reviewed, statistical and economic data were taken, and references describing the investment made in our country. In this context, the government of Ec. Rafael Correa, between 2007 and 2017 progressively increased investment. For the last year in reference, the Secretary of Higher Education, Science and Technology - SENESCYT presented a budget execution of the Higher Education Institutions of USD 1,199 million equivalent to $0.46 \%$ of the Gross Domestic Product, benefiting from a total of 59 universities and polytechnic schools and 241 technical and technological institutes. For the year 2018, the percentage of investment will be affected by the government of President Lenin Moreno, and with the determination to meet the Sustainable Development, Goal 8.2: The highest levels of productivity through technological modernization and innovation, begins In June 2019, the construction of the National Plan for Science, Technology, Innovation and Ancestral Knowledge 2030. To the current date, it can be indicated that one of the results found indicates that our country lacks a philosophical guide and a strategic, integrative programming, generated with the participation of the vital sectors of the country to initiate the required leap in research and development and to promote science, technology and innovation, and the economic growth of the country.

KEYWORDS: science; developing; innovation; investigation; technology.

\section{INTRODUCCIÓN}

La importancia de la ciencia y la tecnología aumenta en la medida en la que el mundo se adentra en lo que se ha dado en llamar "la sociedad del conocimiento", es decir, sociedades en las cuales la importancia del conocimiento crece constantemente por su incorporación a los procesos productivos y de servicios, por su relevancia en el ejercicio de la participación popular en los 

INFORMACIÓN-UNESUM

procesos de gobierno y también para la buena conducción de la vida personal y familiar. La tecnología moderna apoyada en el desarrollo científico (tecnociencia) ejerce una influencia extraordinaria en la vida social en todos sus ámbitos: económico político, militar, cultural. La Revolución Científica del Siglo XVII, y la Revolución Industrial iniciada en el Siglo XVIII fueron procesos relativamente independientes. La fecundación recíproca y sistemática entre ciencia y tecnología es, sobre todo, un fenómeno que se materializa a partir de la segunda mitad del siglo y se acentúa notablemente en el siglo actual. El tránsito que vivimos del siglo XX al siglo XXI es un período profundamente marcado por el desarrollo científico y tecnológico (Nuñez, s.f.).

La Constitución de la República del Ecuador (2008), en su artículo 340, estipula que "El sistema nacional de inclusión y equidad social es el conjunto articulado y coordinado de sistemas, instituciones, políticas, normas, programas y servicios que aseguran el ejercicio, garantía y exigibilidad de los derechos reconocidos en la Constitución y el cumplimiento de los objetivos del régimen de desarrollo. El sistema se articulará al Plan Nacional de Desarrollo y al sistema nacional descentralizado de planificación participativa; se guiará por los principios de universalidad, igualdad, equidad, progresividad, interculturalidad, solidaridad y no discriminación; y funcionará bajo los criterios de calidad, eficiencia, eficacia, transparencia, responsabilidad y participación. El sistema se compone de los ámbitos de la educación, salud, seguridad social, gestión de riesgos, cultura física y deporte, hábitat y vivienda, cultura, comunicación e información, disfrute del tiempo libre, ciencia y tecnología, población, seguridad humana y transporte.

La ciencia y la tecnología en Ecuador es un conjunto de políticas y programas desarrollados por el estado, las asociaciones nacionales e internacionales y las empresas orientada hacia la investigación, el desarrollo y la innovación. La Secretaría de Educación Superior, Ciencia, Tecnología e Innovación - Senescyt, es la encargada de dirigir la política pública en los ámbitos de ciencia, tecnología, innovación y saberes ancestrales; además de coordinar y articular las acciones entre los sectores académico, de investigación, productivos público y privado (Wikipedia, 2019).

En el contexto expuesto, ha sido y será importante reconocer las acciones y proyecciones que realice el estado ecuatoriano para cumplir con las políticas vigentes a través de todas las instituciones públicas y privadas involucradas, sin embargo, también se precisa necesario mostrar resultados cualitativos alcanzados o referencias que muestren los avances del país en el ámbito de la ciencia y tecnología como motor del desarrollo económico y social del Ecuador, en los siguientes apartados se hará una revisión teórica, se mostrará información oficial emitida por los organismos del estado, tanto estadística como económica, referencias filosóficas del tema, apreciaciones de medios públicos y finalmente se mostrarán los resultados percibidos de la presente investigación.

\section{DESARROLLO}

\section{Fundamento filosófico}

Bunge en tanto físico y filósofo autodidacta miró a la Filosofía como "desde afuera", sin ahorrar críticas a teorías, doctrinas y autores en su estilo irreverente. Es así que en "Crisis y reconstrucción de la filosofía" diagnostica un estado de enfermedad disciplinaria y una infertilidad producto del anclaje en el pasado y de la endogamia cultural. Para salir de ese estado Mario propone, además del enfoque sistémico que atraviesa toda su obra, el acercamiento a la ciencia y la tecnología del momento para el abordaje de problemas del mundo real y del conocimiento. También brinda 
Luis Fernando Lucio Villacreses, Miriam Adriana Castillo Merino, Alfredo Lesvel Castro Landin...

algunos indicadores acerca de cómo debería ser la nueva filosofía: auténtica, clara, crítica, profunda, interesante, noble, abierta, realista, sistémica, actualizada y útil.

Es una invitación a los legos a interesarse por un mundo del conocimiento que suena algo incomprensible, alejado de la realidad y bastante inútil. Recuerdo un decano de una facultad de informática al que pregunté si tenían algún estudio sobre filosofía de las ciencias de la información y la comunicación, a lo que contestó que pensaba asomarse a la filosofía al jubilarse, cuando tuviera tiempo libre (Bosch, 2018).

\section{Aproximándose a la ciencia y la tecnología.}

En "Etica y Ciencia y Técnica" se reeditan reflexiones de Bunge sobre las influencias mutuas de las tres disciplinas que datan de más de medio siglo, pero que siguen iluminando con la misma intensidad. Es un buen antecedente antes de encarar el Tomo VIII del Treatise, que más adelante sugerimos.

El azar quiso que "Seudociencia e ideología" cayera en mis manos tempranamente, de manera que pudiera comprender que además de ciencia existe su falsificación (o copia trucha, dirían en Argentina) y que también la ideología juega un rol en las sociedades para bien y para mal. Un texto que nos habla sobre ciencia y tecnología por contraste y que denuncia "mala ciencia", al tiempo que advierte sobre el mal uso de la tecnología. Freud, Marx y Einstein pasan por las páginas de este libro, que además se da espacio para hablar de política, economía, religión, informática y cultura.

El gordo manual de investigación titulado "La investigación científica", es una fuente inagotable de conceptos, métodos y ejercicios prácticos para todo docente investigador o estudiante, sea de ciencias, de ingenierías o de filosofía. Permite meterse de lleno en la cocina científica, desafiando a la búsqueda de la excelencia en investigación y a la conceptualización y tipificación de las actividades en los sistemas de CyT. Es muy útil para despejar muchas confusiones típicas entre ciencia, tecnología e innovación y especialmente indicado para estudiantes de doctorado que se inician en la carrera de investigación. Lamentablemente, la tradición epistemológica y metodológica en nuestro país ignora este texto y recomienda las lecturas de Popper, Feyerabend, Lakatos y Kuhn como si fueran los cuatro mosqueteros de la corte científica.

El aporte y la ubicación social de la ciencia y la tecnología, así como su importancia para el desarrollo nacional se explican muy llanamente en "Ciencia, Técnica y Desarrollo". A dos décadas de aparecido parece más vigente que nunca su argumentación, dado que el neoliberalismo se ha vuelto a adueñar de la Argentina y los primeros pasos para la reducción del sistema científico y tecnológico ya han sido dados, junto con las políticas de apertura de importaciones que fractura una industria nacional incipiente que a su vez demandará menos tecnología. La concepción utilitarista de la ciencia también se reinstala en el poder de la mano de los CEOs que vuelven a colonizar el estado nacional con la regla de la "eficiencia" en la mano (Bunge, 2002).

\section{Estudios filosóficos y sociales de la ciencia y la tecnología}



INFORMACIÓN-UNESUM

La ciencia y la tecnología se han vuelto determinantes del desarrollo económico y cultural de las sociedades actuales, y de ellas depende en forma creciente el bienestar de un país y de sus ciudadanos.

Hoy más que nunca la ciencia y la tecnología están produciendo impactos positivos y negativos en la sociedad y en el ambiente, al tiempo que constituyen uno de los principales recursos para la solución de problemas. A la vez, para su desarrollo dependen de que las sociedades inviertan en ellas sumas considerables de fondos públicos y privados.

Estos procesos han dado lugar a un modelo de sociedad que ha venido imponiéndose en las últimas décadas: la llamada "sociedad del conocimiento", donde la generación de riqueza estaría principalmente basada en el control y el desarrollo de nuevas formas de producción y aprovechamiento del conocimiento.

Uno de los principios rectores de la sociedad del conocimiento es que la ciencia y la tecnología son indispensables para lograr las condiciones materiales, ambientales, sociales y culturales, necesarias para garantizar el bienestar, una vida digna y una organización social justa para las presentes y futuras generaciones. Pero el fortalecimiento y el desarrollo de la ciencia y la tecnología por sí solos no son suficientes, es necesario articular estos sistemas con el resto de la sociedad con el fin de que puedan atenderse los problemas tal y como son percibidos y definidos por los afectados, y de manera que las soluciones sean aceptables para ellos, y no a partir de determinaciones que se hagan parcialmente y al margen de los interesados (UNAM, 2016).

\section{La cibernética de Norbert Wiener}

En el año 1948, Norbert Wiener publicó en Nueva York su Cybernetics, or Control and Communication in the Animal and the Machine (Cibernética o el control y comunicación en animales y máquinas), libro escrito en clave netamente matemática en el que propuso su teoría del control y la comunicación en máquinas y animales, que denominó Cibernética, palabra que hizo

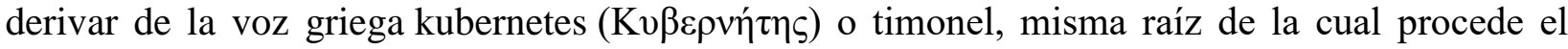
término gobierno y sus derivados.

La Cibernética, nacida de la combinación de las matemáticas y la neurofisiología, se propone como la ciencia que permitirá el control de los "factores antihomeostáticos" inherentes a la Naturaleza y al funcionamiento de la sociedad. La homeostasis es un concepto central de la teoría cibernética, y se refiere al proceso mediante el cual los organismos vivos conservan cierto estado de organización dentro de la tendencia general del universo hacia la corrupción y la decadencia, tendencia que se conoce con el nombre de entropía.

La entropía es la medida de la probabilidad de encontrar respuesta, en un conjunto más grande de universos posibles, a las preguntas que podemos hacernos acerca de algunos de ellos (Wiener 1988a, 14). Dicho más simplemente, la entropía mide el nivel de desorganización y homogeneidad de un determinado sistema (Wiener 1988a, 34). A menor entropía, mayor diferenciación; a mayor entropía, mayor homogeneidad. Wiener creía que a medida que aumentaba la edad del universo aumentaba la entropía y, por extensión, la homogeneidad entre los elementos que lo constituyen. 
Luis Fernando Lucio Villacreses, Miriam Adriana Castillo Merino, Alfredo Lesvel Castro Landin...

La información representa la medida de la reducción de la incertidumbre, esto es, es una medida antientrópica que permite reducir el grado de caos en el Universo. La información se opone a la entropía: a mayor entropía, menor información; a mayor información, menor entropía. Los medios de comunicación (en cuanto que aseguran la libre circulación de la información al interior del medio social) son vistos, en este sentido, como mecanismos fundamentales en la lucha contra la entropía o la tendencia natural a la merma de información en la Naturaleza. Al respecto, Wiener anticipaba, con pocas esperanzas, su preocupación acerca de la propiedad de los medios de comunicación y el uso interesado del poder (ibercienciaoei.org, 2017).

\section{La noción de feedback}

La Cibernética ha colaborado desde sus inicios a formular analogías operativas y funcionales entre hombres y máquinas: de acuerdo con Wiener, el funcionamiento de los seres vivos y el de las máquinas (en particular el de las modernas máquinas electrónicas) son análogos y paralelos en sus tentativas de regular la entropía mediante la retroalimentación. Esta analogía operativa fundamental entre el funcionamiento general de los seres humanos y el de las máquinas se basa en el hecho de que ambos sistemas operan como enclavados locales de entropía negativa, con tendencia temporal creciente hacia mayores niveles de organización (Wiener 1988b, 29-33).

La noción de feedback o retroalimentación es un elemento clave de la teoría cibernética. Se refiere al retorno de salida al interior de un circuito de información, aspecto que habilita la posibilidad de controlar el comportamiento de un sistema, sea técnico, físico, biológico o social. Desde el punto de vista de las teorías de comunicación, eso supone un avance fundamental en cuanto que se reconoce por primera vez la existencia de un esquema circular más complejo y fecundo que supera a las teorías precedentes.

\section{Antecedentes bibliográficos}

Frecuentemente se da un uso indiscriminado de los términos técnica y tecnología, sin embargo, pueden establecerse diferencias, así: la técnica podría ser entendida como un procedimiento para modificar la realidad, basado en la información proporcionada por las ciencias; en cambio, la tecnología podría entenderse como el conjunto de conocimientos acerca del procedimiento técnico o éxito de la acción sobre la realidad.

Cabe explicar que el concepto de técnica quedó precisado por los griegos, de manera especial por Aristóteles, para quien la "téchne (industria, arte, capacidad) es artificiosa y no una actividad natural. La técnica es "saber (como) hacer cosas" según un eidos -idea- que el techenites (industrioso, artesano, artista) posee y que "re-produce" en la realidad (cfr. ibíd.); así lo que era idea de plato en la mente del artesano se convierte en un plato real, apto para servir un alimento mediante la poíesis o acto de producir o fabricar.1 Este recurrir momentáneo a la cultura griega tiene por fin esclarecer el actual concepto de técnica y pretende desmitologizar el vocabulario presente. A través de la historia encontramos que la tecnología surge de la inteligencia 128 del ser humano para responder a sus necesidades esenciales e inmediatas (Fullat, 2000).

La tecnología ha sido entendida de diferentes maneras, unos la conciben como el conjunto de saberes, de habilidades, de destrezas y de medios necesarios para llegar a un fin predeterminado; otros la entienden como un conjunto de conocimientos técnicos, ordenados científicamente, que 

INFORMACIÓN-UNESUM

permiten diseñar y crear bienes y servicios que facilitan la adaptación al medio ambiente y la satisfacción de necesidades y deseos humanos (Aguilar Gordón, 2011).

\section{Función de la tecnología en los nuevos escenarios de la sociedad.}

Se puede avizorar una serie de características independientes de su función principal, de acuerdo al estudio realizado por Marshall McLuhan y B. R. Powers (1994), los objetos tecnológicos cumplen con funciones estéticas y simbólicas. a) Función estética de los objetos tecnológicos. Parte de la creencia del usuario quien busca la belleza a través de las formas, colores y texturas, por ejemplo, en las prendas de vestir, la belleza puede primar sobre las consideraciones prácticas. La ropa es el rubro tecnológico de máxima venta en el planeta porque son la cara que mostramos a las demás personas y condicionan la manera en que nos relacionamos con ellas. b) Función simbólica de los objetos tecnológicos. Cuando la función principal de los objetos tecnológicos es la simbólica, no satisfacen las necesidades básicas de las personas y se convierten en medios para establecer estatus social y relaciones de poder. El caso más destacado y frecuente de 130 objetos tecnológicos fabricados por su función simbólica es el de los grandes edificios: catedrales, palacios, rascacielos gigantes, diseñados para empequeñecer a los que están en su interior, para deslumbrar con exhibiciones de lujo, para infundir asombro, representan símbolo del principal centro del poderío económico (Mcluhan, 1995).

\section{Uso de la tecnología.}

El uso de la tecnología está determinado por la óptica del sujeto que la utiliza y que la valora; en este sentido, desde mi punto de vista se presentan al menos tres aristas de reflexión:

1. Desde la apreciación subjetiva de un hecho (tecnológico).

2. Desde la descripción objetiva de un proceso (tecnológico).

3. Desde los resultados, productos, metas y objetivos alcanzados.

Lo anterior permite inferir que la tecnología puede ser comprendida como proceso observable, lo cual se evidencia en los diversos procesos de formación y de aprendizaje; como sistema, expresada mediante las instituciones sociales; como producto, entendida como resultado de una acción; como proceso de modificación de conductas, reflejada en las actitudes y comportamientos individuales, sociales y culturales en general. Considero que la tarea pendiente para la epistemología de la tecnología es deslindar la definición de fenómeno tecnológico fáctico y la definición de fenómeno tecnológico axiológico. El primero pertenece al ámbito de la necesidad, se vincula con el hecho, con el dato real y observable, por ello es objeto de la ciencia. El segundo pertenece al ámbito de la libertad humana (propia de la moral y de la ética) y por eso mismo, es objeto de la metafísica. Continuando con la reflexión acerca del uso de la tecnología es preciso recalcar que la técnica y la tecnología (instrumento, objeto, hecho) en sí mismas no son buenas ni malas, el uso que el sujeto -ser humano- hace de ella (dependiendo del ejercicio de su libertad) es lo que determina la calidad del juicio (ética).

Podemos sostener que el uso de la técnica y de la tecnología trae consigo ventajas, pero también puede traer desventura para la humanidad; el uso que de ellas se hace es lo que provoca aspectos 
Luis Fernando Lucio Villacreses, Miriam Adriana Castillo Merino, Alfredo Lesvel Castro Landin...

positivos y/o aspectos negativos, que permiten la construcción o la destrucción del ser humano y de su medio ambiente; el uso racional de ellas contribuye para el progreso individual y social.

\section{Los mitos del sistema I + D}

Daniel Sarewitz identifica en 1996 lo que considera como mitos principales del sistema I + D, es decir, de la concepción tradicional de la ciencia y de sus relaciones con la tecnología y la sociedad. Son, en una versión adaptada, los siguientes:

Mito del beneficio infinito: más ciencia y más tecnología conducirá inexorablemente a más beneficios sociales.

$>$ Mito de la investigación sin trabas: cualquier línea razonable de investigación sobre procesos naturales fundamentales es igualmente probable que produzca un beneficio social.

$>$ Mito de la rendición de cuentas: el arbitraje entre pares, la reproducibilidad de los resultados y otros controles de la calidad de la investigación científica dan cuenta suficientemente de las responsabilidades morales e intelectuales en el sistema I + D

$>$ Mito de la autoridad: la investigación científica proporciona una base objetiva para resolver las disputas políticas

$>$ Mito de la frontera sin fin: el nuevo conocimiento científico generado en la frontera de la ciencia es autónomo respecto a sus consecuencias prácticas en la naturaleza y la sociedad (ALONSO \& AYESTARÁN, 1996).

Los estudios y programas sobre Ciencia, Tecnología y Sociedad-CTS se ha desarrollado desde sus inicios en tres grandes direcciones:

$\checkmark$ En el campo de la investigación, los estudios CTS se han planteado como una alternativa a la reflexión académica tradicional sobre la ciencia y la tecnología, promoviendo una nueva visión no esencialista y socialmente contextualizada de la actividad científica.

$\checkmark$ En el campo de la política pública, los estudios CTS han defendido la regulación social de la ciencia y la tecnología, promoviendo la creación de diversos mecanismos democráticos que faciliten la apertura de los procesos de toma de decisiones en cuestiones concernientes a políticas científicas - tecnológicas.

$\checkmark$ En el campo de la educación, esta nueva imagen de la ciencia y la tecnología en la sociedad ha cristalizado la aparición de programas y materias CTS en enseñanza secundaria y universitaria en numerosos países (Gordillo \& Osorio, 2001).

\section{Materiales y métodos.}

\section{Materiales.}



INFORMACIÓN-UNESUM

En el desarrollo de la presente investigación se utilizaron materiales de consulta tanto físicos y digitales que contenían información relevante a la temática abordada.

\section{Método.}

Los métodos utilizados en el presente estudio fueron:

$>$ Método Exploratorio: Este tipo de investigación se centra en analizar e investigar aspectos concretos de la realidad que aún no han sido analizados en profundidad. Básicamente se trata de una exploración o primer acercamiento que permite que investigaciones posteriores puedan dirigirse a un análisis de la temática tratada. Por sus características, este tipo de investigación no parte de teorías muy detalladas, sino que trata de encontrar patrones significativos en los datos que deben ser analizados para, a partir de estos resultados, crear las primeras explicaciones completas sobre lo que ocurre (Castillero Mimenza, 2020).

Método Descriptivo: El objetivo de este tipo de investigación es únicamente establecer una descripción lo más completa posible de un fenómeno, situación o elemento concreto, sin buscar ni causas ni consecuencias de éste. Mide las características y observa la configuración y los procesos que componen los fenómenos, sin pararse a valorarlos. Así pues, en muchas ocasiones este tipo de investigación ni siquiera se pregunta por la causalidad de los fenómenos (es decir, por el "por qué ocurre lo que se observa"). Simplemente, se trata de obtener una imagen esclarecedora del estado de la situación (Castillero Mimenza, 2020).

\section{Resultados}

\section{Evidencia empírica del tema}

En el año 2013 Rosalba Casas realiza un estudio dirigido a 10 países entre ellos Ecuador, indica que desde inicios del Siglo XXI los regímenes políticos que se han venido instaurando, aplican procesos de democratización experimentales, buscan tener un posicionamiento en el tiempo que genera un cambio de discurso respecto a la importancia de ciencia y la tecnología en los proyectos de desarrollo nacional. En este sentido, Mario Bunge quien estuvo en nuestro país en el año 2018 promueve el acercamiento de la ciencia y la tecnología para abordar problemas reales, lo que debe ser tomado como una filosofía autentica, clara, critica, profunda, abierta, realista, sistemática, actualizada y útil.

\section{La apertura de Yachay, salto hacia la sociedad del conocimiento}

"La educación de verdadera calidad es el proyecto más importante del Gobierno" así califica el Presidente de la República Rafael Correa, a uno de los objetivos más importantes de la Revolución Ciudadana; el mejoramiento de la Educación Superior del país, concretado en la creación de cuatro universidades especializadas.

Desde ayer Yachay, la primera universidad de investigación y tecnología del Ecuador, ubicada en el cantón Urcuquí, provincia de Imbabura, dicta los cursos de nivelación a sus primeros 187 
Luis Fernando Lucio Villacreses, Miriam Adriana Castillo Merino, Alfredo Lesvel Castro Landin...

estudiantes, que ahora cumplen su sueño al estudiar en esta universidad. La entidad académica contará con cuatro zonas; dos agrícolas, una urbana y una tecnológica.

Durante el discurso de inauguración, Daniela Armijo, una de las estudiantes, agradeció al Presidente "por devolvernos la esperanza de un nuevo Ecuador", palabras que llenaron de emoción a todos los presentes en especial al Primer Mandatario y René Ramírez, Secretario de Educación Superior, Ciencia, Tecnología e Innovación, quien en su intervención aseguró que no podía agregar más palabras a las ya mencionadas por Daniela.

Por su parte, el presidente Correa destacó que la apertura de Yachay es el primer paso hacia la sociedad del conocimiento y reiteró que "la ciencia, la tecnología y la innovación son fundamentales para el desarrollo... creo en el poder transformador de la ciencia y la tecnología. En ese poder deposito gran parte de mi esperanza en el futuro del planeta, en la sostenibilidad de nuestro modo de vida, en la posibilidad de alcanzar el buen vivir para toda la humanidad".

Rafael Correa, señaló la inmensa responsabilidad que tiene sobre sus hombros el gobierno, el dinero que antes se desperdiciaba, hoy es administrado por manos honestas con capacidad e inteligencia. "Si algo hay que agradecer es a sus familias y al pueblo ecuatoriano, y la mejor manera de hacerlo es trabajar con excelencia, con integridad, con capacidad con esfuerzo, ¡cómo los envidiamos queridos jóvenes, felicitaciones! Ustedes son 187 promesas para el futuro de la Patria, tendrán 500 horas de nivelación en las materias de Física, Biología y Matemática, dictadas por 40 docentes nacionales y extranjeros, todos con PHD; equivalente al máximo nivel académico de las mejores universidades del mundo, lo que se decía que era imposible, se está demostrando que es perfectamente posible: tener un núcleo de docentes de nivel mundial."

\section{Resumen ejecutivo de las inversiones realizadas por el estado ecuatoriano (Senescyt, 2018).}

La Secretaría de Educación Superior, Ciencia, Tecnología e Innovación para año 2017 presentó una ejecución presupuestaria de las IES públicas de 1.199 millones de USD y un gasto corriente ejecutado de 59,92 millones de USD. En el registro de instituciones de educación superior en el caso de universidades y escuelas politécnicas para el año 2018 se registró un total de 59 universidades y escuelas politécnicas y 241 institutos técnicos y tecnológicos.

El registro de matrícula durante el período 2012-2016 en universidades y escuelas politécnicas creció de forma acumulada en alrededor de 17\%, que para el año 2016 se registraron 594.106. De este total el $58 \%$ correspondían a instituciones públicas. Las carreras con más altos niveles de matrícula son ciencias sociales, educación comercial y derecho con 267.338 registros, de las cuales el 58\% son elegidas por mujeres, seguidas por ingenierías con 85.361 en donde el $75 \%$ son elegidas por hombres y carreras de salud y servicios con 83.530 registros, en donde el $67 \%$ son mujeres.

La participación de docentes por género se presenta un $61 \%$ de hombres, mientras que la participación de mujeres asciende a 39\%. Además, el $60 \%$ de los docentes trabajan bajo relación de dependencia, seguidos por aquellos que tienen nombramiento $27 \%$. Finalmente, los docentes con título de PhD se han duplicado en el período 2012-2016 que pasan de 1.056 a 2.776.

La SENESCYT al 05 de diciembre de 2018 registra 2.112.835 títulos. Del total de títulos registrados $87 \%$ son nacionales y $13 \%$ son extranjeros. 

INFORMACIÓN-UNESUM

De acuerdo con datos de la SENESCYT acumulado al año 2018 se registró un total de 16.406 becas nacionales y 14.133 becas internacionales y cooperantes.

\section{Inversiones realizadas}

Según datos de la Secretaría Nacional de Ciencia, Tecnología e Innovación para el año 2017 la ejecución presupuestaria de las IES asciende a 1.199 millones, lo que significa un incremento del $6,1 \%$ con relación al 2016.

Tabla 1. Proyectos de inversión en millones de dólares

\begin{tabular}{|c|r|c|}
\hline Año & $\begin{array}{r}\text { Ejecución IES } \\
\text { públicas }\end{array}$ & Var.\% YoY \\
\hline 2009 & 726 & \\
\hline 2010 & 856 & $17,84 \%$ \\
\hline 2011 & 864 & $0,90 \%$ \\
\hline 2012 & 881 & $1,97 \%$ \\
\hline 2013 & 980 & $11,28 \%$ \\
\hline 2014 & 1.084 & $10,60 \%$ \\
\hline 2015 & 1.192 & $9,98 \%$ \\
\hline 2016 & 1.130 & $-5,18 \%$ \\
\hline 2017 & 1.199 & $6,07 \%$ \\
\hline
\end{tabular}

Fuente: Boletín informativo de la Senescyt.

Gráfico 1. Ejecución presupuestaria de las Instituciones de Educación Superior del Ecuador en millones de dólares. 


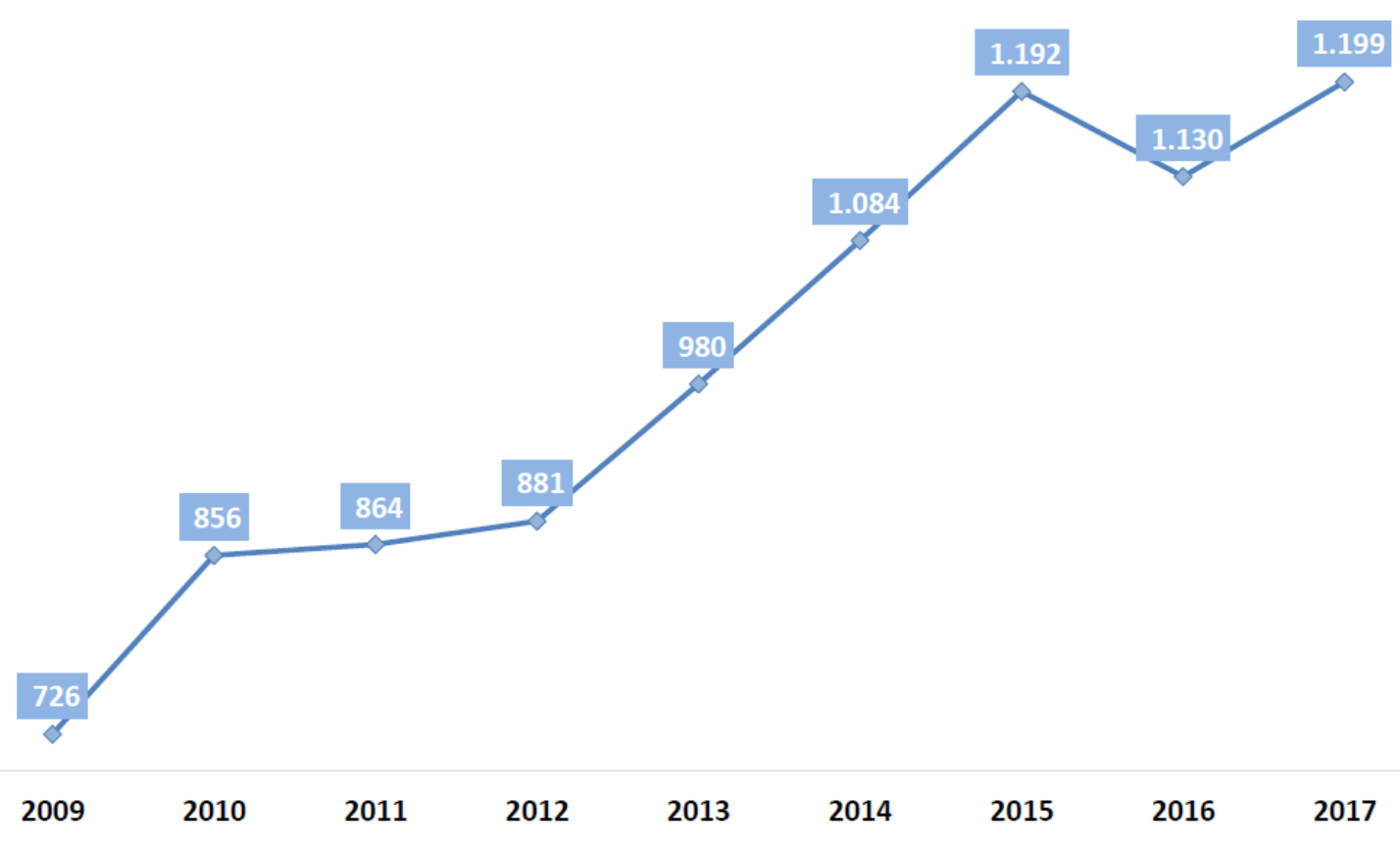

Fuente: Boletín informativo de la Senescyt.

Grafico. 2 Gasto I+D como (\%) del PIB

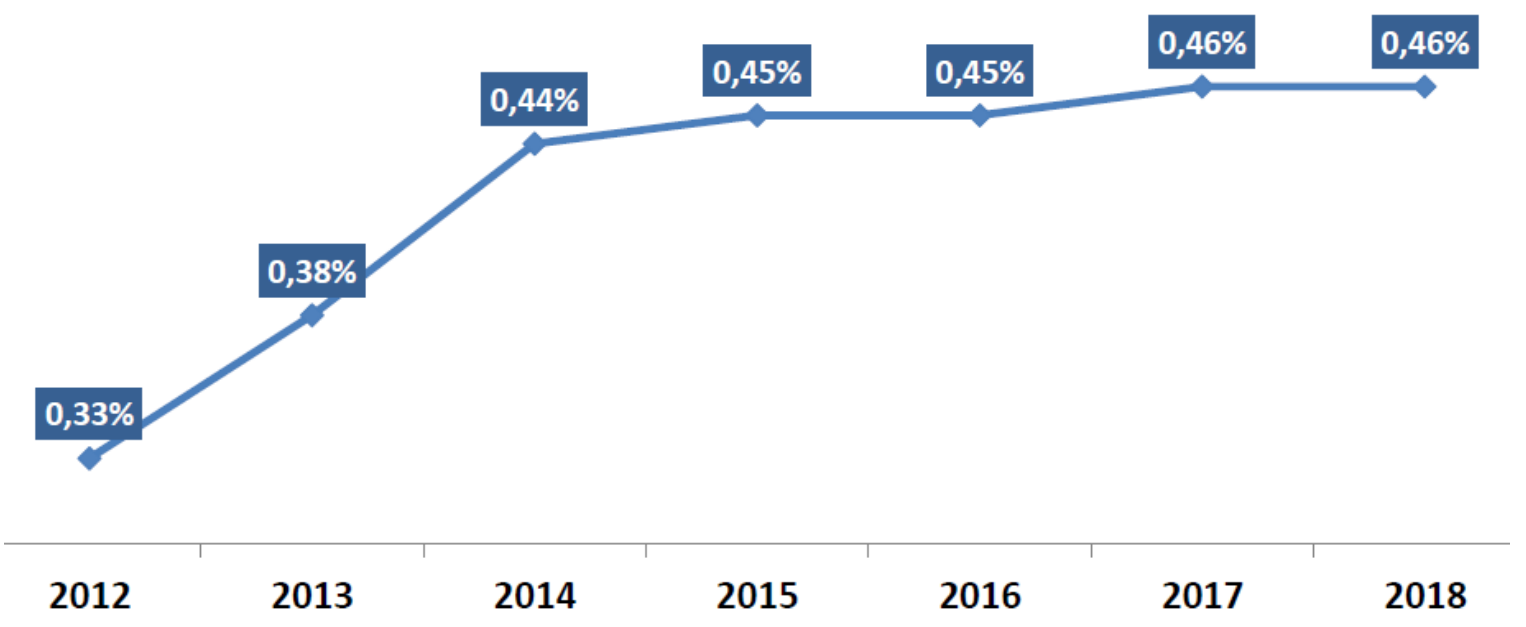

Fuente: Boletín informativo de la Senescyt.

Tabla 2. Campo de estudios según género

114 UNESUM-Ciencias. Publicación cuatrimestral. Vol. 4, Año 2020, No. 1 (Enero - Abril) 
VIVENCIAS ACADÉMICAS DE LOS ESTUDIANTES DE I SEMESTRE MATUTINO DE TECNOLOGÍA DE LA INFORMACIÓN-UNESUM

\begin{tabular}{|l|r|r|r}
\multicolumn{1}{c|}{ Campo de estudios } & \multicolumn{1}{c|}{ Hombre } & \multicolumn{1}{c|}{ Mujer } & \multicolumn{1}{c}{ Total } \\
\hline Total & $\mathbf{2 8 2 . 4 3 8}$ & $\mathbf{3 1 1 . 6 6 8}$ & $\mathbf{5 9 4 . 1 0 6}$ \\
\hline Ciencias sociales, educación comercial y derecho & 110.413 & 156.925 & 267.338 \\
\hline Ciencias & 108.379 & 122.279 & 230.658 \\
\hline Ingeniería, industria y construcción & 64.349 & 21.012 & 85.361 \\
\hline Salud y servicios & 27.559 & 55.971 & 83.530 \\
\hline Educación & 11.867 & 24.743 & 36.610 \\
\hline Agricultura & 14.043 & 12.611 & 26.654 \\
\hline Humanidades y artes & 10.414 & 8.302 & 18.716 \\
\hline Servicios & 6.270 & 8.603 & 14.873 \\
\hline Programas y certificaciones genéricos & 38 & 81 & 119 \\
\hline
\end{tabular}

Fuente: Boletín informativo de la Senescyt.

Tabla 3. Nivel de estudios

\begin{tabular}{|l|r|r|r|r|}
\hline Nivel de estudios & $\begin{array}{c}\text { Acumulado a } \\
2015\end{array}$ & 2016 & 2017 & A octubre 2018 \\
\hline Total & $\mathbf{1 1 . 4 6 9}$ & $\mathbf{5 5 8}$ & $\mathbf{7 8 9}$ & 496 \\
\hline $\begin{array}{l}\text { Doctorado y post } \\
\text { doctorado }\end{array}$ & 1.668 & 32 & 153 & 94 \\
\hline $\begin{array}{l}\text { Especialidades } \\
\text { médicas }\end{array}$ & 1.302 & 12 & 12 & 48 \\
\hline $\begin{array}{l}\text { Investigación/ } \\
\text { docencia }\end{array}$ & 21 & 8 & 7 & - \\
\hline Maestría & 2.538 & 196 & 462 & 255 \\
\hline Pregrado & 2.408 & 302 & 153 & 99 \\
\hline Otros & 1.532 & 8 & 2 & - \\
\hline
\end{tabular}

Fuente: Boletín informativo de la Senescyt.

Rendición de cuentas, Yachay 2018.

Uno de los objetivos de la universidad del conocimiento señala: Procurar que la Ciudad del Conocimiento Yachay sea un territorio de generación de conocimiento para el país. La Empresa 
Luis Fernando Lucio Villacreses, Miriam Adriana Castillo Merino, Alfredo Lesvel Castro Landin...

Pública Yachay E.P. enfoca todos los esfuerzos en impulsar, planificar, coordinar y ejecutar programas y proyectos de educación, arte y cultura, gestión del conocimiento, investigación científica, desarrollo tecnológico, transferencia de tecnología, propiedad intelectual, y la formación de talento humano altamente capacitado en el país.

\section{Principales resultados del informe preliminar presentado en el 2018:}

$\checkmark$ En el transcurso del año 2018 se tuvo un alcance de 410 personas capacitadas en emprendimiento e innovación.

$\checkmark 5$ asesorías a empresas en diferentes temáticas de innovación.

$\checkmark$ De 22 proyectos de emprendimiento o startups, 13 se encuentran en proceso de incubación, 3 culminan los procesos y 6 continuaran para el 2019.

$\checkmark \quad 10$ emprendimientos de base tecnológica que ingresaron firmando convenio PIEBT (Programa de Incubación de Emprendimientos de Base Tecnológica), superan procesos de incubación. 3 publicaciones científicas mediante el uso del Supercomputador Quinde I - HPC.

$\checkmark 21$ proyectos (ofertas de cursos de capacitación) atendidos en el Centro de Desagregación Tecnológica - CDT en Ingeniería Inversa, Prototipado y Mecanizado.

Se desarrolló el catálogo de productos y servicios, obteniendo ya el documento final y material promocional por cada línea de negocio definida esto de forma conjunta entre los Agregadores de Valor, Planificación, Comercial y Comunicación: - Parque Científico Tecnológico Industrial - Proyectos Tecnoagrícolas - Otros Productos y Servicios Ejecución del primer programa de Innovación Coreano Ecuatoriano entre la Empresa Pública Yachay EP y KOICA, el evento Pitch Day, permitió a 13 startups a nivel nacional presentar sus proyectos a expertos y potenciales inversionistas de Corea del Sur

\section{Agenda 2030 de Desarrollo Sostenible.}

Nuestro país está comprometido con la inclusión de políticas públicas que aporten al cumplimiento de la Agenda 2030 de Desarrollo Sostenible, siendo enfático la observancia por lo menos del siguiente objetivo y meta:

$\checkmark$ Objetivo 8: Promover el crecimiento económico sostenido, inclusivo, el empleo pleno y productivo y el trabajo decente para todos.

- Meta 8.2: Lograr niveles más elevados de productividad mediante la modernización tecnológica y la innovación.

\section{La realidad tecnológica de Ecuador (Teleamazonas, 2019).}

Expertos nacionales e internacionales en materia de invernaderos científicos debaten estos días en Quito sobre la necesidad de que Ecuador avance en materia de innovación tecnológica, sobre todo 

INFORMACIÓN-UNESUM

cuando se encuentra a 20 años de distancia de los países de su entorno. En esta idea coincidieron varios expertos durante la inauguración del simposio «Parques científicos y desarrollo local», que este lunes y martes tiene lugar en la Escuela Politécnica Nacional (EPN), en donde también se ha destacado que el país tiene que desarrollarse en la construcción de sinergias y estrategias mancomunadas entre los distintos actores.

Todo ello, como apuntó Adriana Arango, encargada del programa de Medicina de la Universidad Remington y profesora de la Universidad de Antioquia (Colombia), bajo la premisa de que estos conocimientos tienen que tener en cuenta las necesidades de la población. «Hay que pensar en las personas, que son las que van a utilizar todo este conocimiento», afirmó a Efe para después añadir que es necesario conocer «mucho el medio» en el que se mueven las investigaciones y haberlo «vivido».

Ecuador, cuya Constitución de 2008 introdujo la necesidad de crear un sistema nacional de ciencia, tecnología e innovación que fomente la investigación y el desarrollo tecnológico en articulación con el sector productivo, todavía no ha dado el «salto», según los expertos, hacia la tercera fase del desarrollo científico-tecnológico.

Esta es lograr que la investigación que se genera en las universidades se convierta en transferencia de conocimiento, por lo que dé respuesta a problemas sociales con una mirada hacia la comunidad que demanda esos conocimientos. Según la coordinadora del Grupo de Investigación Multidisciplinar en Sistemas de Información, Gestión de la Tecnología e Innovación, Valentina Ramos, «Ecuador ha llegado tarde a la definición de estrategias mancomunadas para el crecimiento regional» en lo que a ciencia se refiere. Es algo en lo que convinieron representantes de los tres actores del desarrollo científico: academia, Estado y sector productivo.

Como indicó el director del Centro de Apoyo al Desenvolvimiento Científico y Tecnológico de la Pontificia Universidad Católica de Río Grande do Sul (Brasil), Eduardo Giugliani, es necesario articular «una estrategia de innovación en conjunto», ya que es lo que buscan las propias empresas.

«Las empresas buscan hoy espacios como estos, en ecosistema, para tener conexiones en red. Para desarrollar el conocimiento de hoy es necesario tener conexiones transversales», explicó a Efe. Por su parte, el subdecano de la Facultad de Ciencias Administrativas de la EPN, Antonio Franco, destacó que Ecuador necesita cambiar «drásticamente» porque, si no, un futuro «drástico, complejo y oscuro» podría darse.

Esto es así porque tachó al país de ser «muy débil» en cuanto a cuestiones políticas, económicas y sociales, por lo que problemas graves que ya son una realidad -como la migración o el cambio climático- no encontrarán una respuesta de Ecuador para hacerles frente. Y puso como ejemplo el número de patentes que el país presentó ante organismos internacionales durante 2015: 140, es decir, 30 veces menos que las que desarrolló Colombia. De ellas, solo el $10 \%$ eran de ciudadanos ecuatorianos, lo que representa entre 15 y 20 patentes del total.

Con todo, para Giugliani el número de patentes no debería ser «el principal indicador» para que se pueda hablar de «una nueva trayectoria de desarrollo para la sociedad», y llamó a tener en cuenta la sustentabilidad y competitividad de las empresas. 
Luis Fernando Lucio Villacreses, Miriam Adriana Castillo Merino, Alfredo Lesvel Castro Landin...

Los expertos también comentaron que Ecuador tiene un problema en cuanto a la inversión: solo se dedica a investigación y desarrollo el 0,4 \% del PIB, por el 2,4 \% de media que dedican los países de la Organización para la Cooperación y el Desarrollo Económicos (OCDE).

Igualmente, existe un problema en cuanto al porcentaje de esa inversión que recae sobre el Estado: el $63 \%$, por el $33 \%$ que viene de las Universidades, lo que tampoco se refleja entre los países de la OCDE -de media, los Estados financian el $35 \%$ de esas inversiones.

\section{Senescyt inicia la construcción del Plan Nacional de Ciencia, Tecnología, Innovación y Saberes Ancestrales 2030}

Implementar, promover y fomentar la investigación, el desarrollo tecnológico y la innovación en el país es prioridad para la Secretaría de Educación Superior, Ciencia, Tecnología e Innovación (Senescyt), y en el marco de este esfuerzo, construir el Plan Nacional de Ciencia, Tecnología, Innovación y Saberes Ancestrales 2030. Este plan estratégico, que espera definir acciones a corto, mediano y largo plazo, incluirá la participación activa de investigadores, innovadores, estudiantes, emprendedores, docentes de universidades, de institutos tecnológicos y otros actores sociales y estatales.

En ese marco, la Senescyt se reunió con representantes de 11 institutos públicos de investigación para escuchar sus aportes y así crear de manera conjunta este insumo que contribuirá al desarrollo social y económico del país. Entre tanto, Karla Tinoco, directora del Instituto Nacional de Investigaciones Agropecuarias, resaltó el interés de la Senescyt alrededor de la ciencia, tecnología e innovación. "La creación del Plan no solo nos marca el camino a seguir, sino que dota de relevancia a este tema que muchas veces está olvidado".

Por medio del Plan, se aspira impulsar la investigación científica, tecnológica y la innovación orientada a la solución de problemas y a la satisfacción de demandas en áreas estratégicas para Ecuador, de aquí al 2030, además, fomentar la sinergia entre el sector público, privado y la academia. También se quiere expandir el acceso y difusión del conocimiento, fomentando su transferencia y apropiación social y colectiva; y, fortalecer las herramientas de los investigadores en materia de propiedad intelectual con miras a estimular la competitividad en productos nacionales en el mercado nacional e internacional (Senescyt, www.educacionsuperior.gob.ec, 2019).

\section{Financiamiento para investigación y desarrollo es limitado en Ecuador}

El último informe sobre Ecuador de la Organización Mundial de la Propiedad Intelectual (OMPI), que mide el nivel de innovación de los países, resalta aspectos positivos como una mejor infraestructura, pero indica también como negativo el reducido número de patentes que se registran y la nula inversión en capitales de riesgo. Destinar más fondos para investigación y desarrollo es una necesidad. Según los últimos datos reportados al Banco Mundial, 0,44 \% del Producto Interno Bruto (PIB) del país se destina con esos fines. En otros países de América Latina como México es 0,49 \%; Argentina, 0,53 \%; y Brasil, 1,2\%. La diferencia es abismal con las economías más desarrolladas, como Corea del Sur que destina 4,23 \% del PIB; Israel, 4,25 \%; Japón, 3,14 \%; Alemania, 2,9 \%; Estados Unidos, 2,7 \%; y China 2,11 \%. Sin embargo, cada vez hay más publicaciones científicas del país en revistas indexadas. 
Gráfico 3. Ranking según el índice de innovación global 2019. Posiciones de países de América Latina y el Caribe

51 Chile

55 Costa Rica

56 México

62 Uruguay

66 Brasil

67 Colombia

69 Perú

73 Argentina

75 Panamá

81 Jamaica

87 República Dominicana

91 Trinidad y Tobago

95 Paraguay

99 Ecuador

104 Honduras

107 Guatemala

108 El Salvador

110 Bolivia

\section{Nicaragua}

Fuente: Organización Mundial de Propiedad Intelectual

EL UNIVERSO

\section{Fuente: Diario el Universo}

\section{CONCLUSIONES}

Ecuador ha tenido la presencia de ilustres maestros de la filosofía como Mario Bunge y Rosalba Casas, quienes opinaron sobre la implementación de la ciencia y la tecnología en los países en desarrollo, sin embargo, no se aprecia en nuestro país la definición de una corriente epistemológica clara, que motive el desarrollo científico y tecnológico basado en la solución de los problemas más urgentes, que repercuta en su crecimiento económico. 
Luis Fernando Lucio Villacreses, Miriam Adriana Castillo Merino, Alfredo Lesvel Castro Landin...

Es notorio que desde el aparecimiento del gobierno del Ec. Rafael Correa, entre los años 2007 2017 se fue incrementando progresivamente la inversión en I + D, lográndose inicialmente que todas las universidades del país reciban ingentes cantidades de dinero para promover el desarrollo de la ciencia y la tecnología, sin embargo los resultados más relevantes giran en torno a la formación profesional de cuarto y quinto nivel no siendo necesariamente un indicador de desarrollo país vinculado a generar avances o descubrimientos que nos ubique en sitiales relevantes del quehacer científico internacional.

La inversión más notoria realizada en el Ecuador fue la implementación de la universidad del conocimiento "Yachay" que ha generado expectativas en nuestro país y en todos los rincones donde el Ex presidente Correa le visibilizó, sin embargo, el informe emitido por la empresa pública creada para su implementación y administración, muestra resultados preliminares que no datan de mostrar resultados científicos que se liguen a la solución de los problemas locales.

El actual gobierno nacional liderado por el Lcdo. Lenin Moreno en vista de los avances logrados a la fecha, inició en el presente año, bajo el liderazgo de la Secretaria Nacional de Ciencia, Tecnología y Saberes Ancestrales, la elaboración del Plan Nacional 2030, y hasta la presente fecha no se cuenta con un documento terminado y/o promulgado oficialmente para su implementación. Lo expuesto, también denota la ausencia de una guía filosófica y una programación estratégica, integradora, generada con la participación de los sectores vitales del país para iniciar el salto requerido en I + D y promover ciencia, tecnología e innovación, y el crecimiento económico del país. Esto último aporta significativamente al cumplimiento de la agenda 2030 de Desarrollo Sostenible y es un compromiso a desarrollar por el estado ecuatoriano.

A la emisión del presente documento no existe evidencia pública de las inversiones realizadas por el actual gobierno o al menos esta información no pudo encontrarse tras la búsqueda realizada, sin embargo el estudio realizado por la Organización Mundial de la Propiedad Intelectual (OMPI) determina que nuestro país se encuentra por debajo de Chile, Brasil, Colombia y otros países de América Latina y el Caribe, ubicándose en el puesto 99, urgiendo la necesidad de que el gobierno de turno destine más fondos para investigación y desarrollo.

\section{REFERÉNCIAS BIBLIOGRÁFICAS}

Aguilar Gordón, F. (2011). REFLEXIONES FILOSÓFICAS SOBRE LA TECNOLOGÍA Y SUS NUEVOS ESCENARIOS. Dialnet_unirioja.es, 124-174.

ALONSO, A., \& AYESTARÁN, I. y. (1996). Ciencia, Tecnología y Sociedad: una aproximación conceptual. Cuadernos de Iberoamérica.

Bosch, M. (Agosto de 2018). Filosofía de la ciencia y la Tecnología 1 Mario Bunge: Inagotable pensador sistémico Inagotable pensador sistémico. Obtenido de Instituto Nacional de Tecnología Agropecuaria: https://www.researchgate.net/publication/326922331_Filosofia_de_la_ciencia_y_la_Tecnologia_1_Mario_ Bunge_Inagotable_pensador_sistemico_Inagotable_pensador_sistemico

Bunge, M. (2002). Filosofia de la Ciencia y la Tecnología. Siglo XXI Editores.

Castillero Mimenza, O. (2020). https://psicologiaymente.com. Obtenido de https://psicologiaymente.com/miscelanea/tipos-de-investigacion

Fullat, C. (2000). El positivismo de Comte. 164.

Gordillo, M., \& Osorio, C. (2001). Ciencia, tecnología y sociedad: Una aproximación conceptual. Apuntes de Iberoamérica.

ibercienciaoei.org. (2017). La cibernética de Norbert Wiener. Por un uso ético de la tecnología.

Mcluhan, M. (1995). Las redes sociales y la Aldea Global - Dialnet. Obtenido de https://dialnet.unirioja.es: https://dialnet.unirioja.es > descarga $>$ articulo

Nuñez, J. (s.f.). https://www.oei.es. Obtenido de https://www.oei.es/historico/salactsi/nunez05.htm 
UNESUM-Ciencias: Revista Científica Multidisciplinaria

ISSN 2602-8166

VIVENCIAS ACADÉMICAS DE LOS ESTUDIANTES DE I SEMESTRE MATUTINO DE TECNOLOGÍA DE LA INFORMACIÓN-UNESUM

Senescyt. (2018). Boletín analítico de educación superior, ciencia, tecnología, innovación y saberes ancestrales.

Senescyt. (7 de junio de 2019). Obtenido de www.educacionsuperior.gob.ec: https://www.educacionsuperior.gob.ec/senescyt-inicia-la-construccion-del-plan-nacional-de-cienciatecnologia-innovacion-y-saberes-ancestrales-2030/

Teleamazonas. (27 de 05 de 2019). www.teleamazonas.com. Obtenido de http://www.teleamazonas.com/2019/05/larealidad-tecnologica-de-ecuador/

UNAM. (2016). Universidad Nacional Autónoma de México . Obtenido de http://www.posgrado.unam.mx/filosofiadelaciencia/programa/campos/estudios-filosoficos-sociales-cienciatecnologia.html

Wikipedia. (11 de Octubre de 2019). https://es.wikipedia.org. Obtenido de https://es.wikipedia.org/wiki/Ciencia_y_tecnología_en_Ecuador. 
Luis Fernando Lucio Villacreses, Miriam Adriana Castillo Merino, Alfredo Lesvel Castro Landin...

122 UNESUM-Ciencias. Publicación cuatrimestral. Vol. 4, Año 2020, No. 1 (Enero - Abril) 